\title{
Plasma Nesfatin-1 Levels and its Relationship with Anxiety Levels in Obesity Diagnosed Population: A Controlled Study
}

\author{
Hayriye Baykan ${ }^{1}$ (D) Ozgur Baykan² (D), Onur Durmaz ${ }^{3}$ (D) , Oguz Elif Gulsah ${ }^{1}$ (D) , Hayrettin Kara ${ }^{4}$, Serap Akdeniz Gorgulu ${ }^{1}$ (D) \\ Ali Yaman ${ }^{5}$ (D), Tunay Karlidere ${ }^{1}$ (D) \\ ${ }^{1}$ Balikesir University, Department of Psychiatry, Balikesir, Turkey \\ ${ }^{2}$ Balikesir Ataturk State Hospital, Biochemistry, Balikesir, Turkey \\ ${ }^{3}$ Erenkoy Mental Health and Neurology Training \& Research Hospital, Psychiatry, Istanbul, Turkey \\ ${ }^{4}$ Balikesir University Health Practice and Research Hospital, Nutrition and Dietetic, Balikesir, Turkey \\ ${ }^{5}$ Balikesir University Faculty of Medicine, Department of Biochemistry, Balikesir, Turkey \\ Correspondence Author: Hayriye Baykan \\ E-mail: hayriyebaykan@gmail.com \\ Received: $17.02 .2018 \quad$ Accepted: 02.05 .2018
}

\begin{abstract}
Objective: Nesfatin-1 is a satiety neuropeptide involved in the regulation of metabolic pathways and food intake. Some studies have shown nesfatin-1 to be also associated with stress responses and stress-related behaviors. In the present study, we evaluated nesfatin-1 levels in obese individuals and investigated whether nesfatin-1 levels could be associated with anxiety levels in obese populations.

Methods: Fifty-eight obese ( 29 anxious and 29 non-anxious) patients and 25 healthy control subjects between 18 and 65 years old were enrolled in the study. Plasma nesfatin-1 levels were measured with a commercial enzyme-linked immunosorbent assay, and anxiety levels were measured using the Hospital Anxiety and Depression Scale.

Results: Plasma nesfatin-1 levels were significantly lower in both obese and anxious subjects and obese and non-anxious subjects than in the control group. No significant differences were found between the obese+anxious group and the obese+nonanxious group.

Conclusion: Our results suggest that plasma nesfatin-1 levels are decreased and not associated with anxiety levels in obese populations Keywords: Nesfatin-1, Obesity, Anxiety
\end{abstract}

\section{INTRODUCTION}

Nesfatin-1 is a multifunctional peptide with pleiotropic functions secreting from different systems. The most established functions associated with nesfatin- 1 have been reported as anorexigenic effect, feed regulation, and appetite (1). Studies on animals showed that nesfatin-1 is derived from nucleobindin-2 and is expressed in several regions of the central nervous system (CNS), including the limbic system, brainstem, and hypothalamus (2). Several studies showed that nesfatin-1-secreting neurons are distributed in the regions of the CNS associated with neurovegetative functions, such as food intake regulation (3). Some data revealed that nesfatin-1 is expressed in the regions other than the CNS in the human body, including adipose tissue, and has potential regulatory functions in association with some other metabolic hormones, such as ghrelin (4). There is also evidence supporting that nesfatin-1 is implicated in adipogenesis and obesity (5). Obesity and metabolic syndrome, as well as diabetes and cardiovascular diseases, have been associated with the dysfunction of nesfatin-1 and its associated pathways (6). Nesfatin-1 levels have been reported to be increased in obese individuals, whereas they have been found to be decreased in cases with anorexia nervosa (7). In line with this data, a positive correlation between nesfatin-1 levels and body mass index (BMI) has been observed in some studies, whereas some conflicting data regarding a negative correlation between nesfatin-1 levels and $\mathrm{BMI}$ also exist (8). There is evidence of low concentrations of nesfatin-1 in populations with high BMI. A previous study showed a negative correlation between BMI and nesfatin-1 in non-obese populations (8). Nesfatin-1 has been reported to be expressed in several hypothalamic nuclei and regions associated with appetite and emotional status (9). Furthermore, central regulatory neuropeptides, such as neuropeptide $Y$, vasopressin, and oxytocin, that contribute to energy balance are expressed from neurons in the arcuate, paraventricular, and supraoptic hypothalamic nuclei similar to nesfatin-1. Although a specific nesfatin-1 receptor is still unknown, current data showed a proposed mechanism of action that involves nesfatin-1 interacting with a $\mathrm{G}$ proteincoupled receptor that results in an inhibitory hyperpolarization of neuropeptide Y/agouti-related peptide neurons that have an orexigenic effect in the arcuate nucleus (10). In addition, nesfatin-1 has been reported to stimulate oxytocin-mediated melanocortin that has an anorexigenic effect in the paraventricular nucleus $(10,11)$. Neurovegetative functions, including appetite and sleep, as well as some other hypothalamic 
functions, are related to and influenced by an individual's emotional state. As nesfatin-1 is a satiety neuropeptide involved in the regulation of metabolic pathways and food intake, some studies showed that nesfatin- 1 is also associated with stress responses and stress-related behaviors $(7,12)$. A previous study conducted on rats showed that intracerebroventricular nesfatin-1 injections yield anxiety - and fear-related behaviors (9). Data on the association between nesfatin-1 and stress responses in human subjects are limited. However, in recent years, nesfatin-1 has been studied in subjects with psychiatric disorders, such as depression and anxiety in particular. Some studies found a relationship between nesfatin-1 levels and anxiety disorders, such as panic disorder, generalized anxiety disorder, and obsessive-compulsive disorder $(13,14,15)$. In a previous study, nesfatin-1 levels were found to berelatively higher in patients diagnosed with panic disorder than in the control group (13). However, data regarding nesfatin-1 levels in patients diagnosed with generalized anxiety disorder are conflicting $(15,16)$, and studies examining nesfatin-1 levels in subjects with affective disorders are limited. One study showed nesfatin-1 levels to be significantly higher in subjects with major depression than in the control group, whereas another study reported decreased nesfatin-1 levels during manic episodes $(17,18)$. All of this data supports nesfatin-1 as a central neuropeptide participating in metabolic regulation, emotional state, and stress responses. To our knowledge, although nesfatin-1 levels have been studied in relation to obesity and some psychiatric disorders, such as depression and anxiety, data on the association between nesfatin-1 levels and anxiety in obese populations are limited. In the present study, we evaluated nesfatin-1 levels in obese individuals and investigated whether nesfatin-1 levels are associated with anxiety levels in obese populations.

\section{METHODS}

The study was performed in accordance with the Declaration of Helsinki. The local ethics committee of Balıkesir University Faculty of Medicine approved the study (2014/04). Written informed consent was obtained from all the participants in the study. Fifty-eight obese patients and 25 healthy controls (non-obese and non-anxious) between 18 and 65 years old were enrolled in the present study. Patients were divided into two groups based on whether they had comorbid anxiety ( 29 obese and anxious and 29 obese and non-anxious). Patients were selected from those who applied to the psychiatry outpatient clinic and were diagnosed with anxiety disorder or from those who were directed to the psychiatry outpatient clinic due to their psychiatric complaints from the dietary outpatient clinic. All patients were diagnosed with obesity with BMIs measuring $>30 \mathrm{~kg} / \mathrm{m} 2$. Diagnosis of obesity was made after $\mathrm{BMI}$ was measured by a dietitian. A psychiatric evaluation and diagnosis were made by a psychiatrist. Patients met the criteria for anxiety disorders, as confirmed by the Structured Clinical Interview for Diagnostic and Statistical Manual of Mental Disorders, 4th Edition Axis I Disorders (SCID-I) (19). The control group was selected from individuals who had no psychiatric diagnoses, as confirmed by the SCID-I, as well as no obesity confirmed by BMI measurements $<25 \mathrm{~kg} / \mathrm{m} 2$.The Hospital Anxiety and Depression Scale (HADS) was used to determine the severity of their anxiety (20). The HADS was developed to detect anxiety and depression levels in outpatient clinic settings. The Turkish version of the HADS was erformed by Aydemir et al.with a cut-off value of 10 for anxiety to be used in the Turkish version (21). Patients with psychiatric or neurological diagnoses other than anxiety disorders, such as mental retardation, alcohol/substance use disorder, history of electroconvulsive therapy, history of psychiatric drug use in the last 3 months, a severe physical illness, such as diabetes or another endocrinopathy, cardiovascular diseases, malignity, infectious diseases, and pregnancy, were excluded from the study. Patients were selected, instructed about the study, and handed written informed consent on their first visit. Venous blood samples wereobtained between 07:00 and 08:00 AM in the morning of the second admission after an overnight fasting period. All blood samples were collected in standard ethylenediaminetetraacetic acid tubes (Becton, Dickinson, USA). After blood was withdrawn, plasma samples were centrifuged at $1300 \times \mathrm{g}$ for $10 \mathrm{~min}$. Plasma samples were placed into tubes, and $1 \%$ concentrated protease inhibitor cocktail (Sigma Aldrich, USA) was administrated in all tubes. Samples were stored at $-20{ }^{\circ} \mathrm{C}$ until further processing. Nesfatin-1 levels were measured using a commercial enzyme-linked immunosorbent assay kit (ELISA, RayBiotech, USA). All samples were processed in one batch without dilution. Intra-assay and inter-assay variabilities were $<10 \%$ and $<15 \%$, respectively, according to the manufacturer.

\subsection{Statistical Analysis}

Data were analyzed using the SPSS software version 15.0 (IBM Inc., Chicago, IL, USA). The ANOVA test was used to analyze the differences between mean values of the groups when normally distributed variables are homogenous. The Tukey test was used for post hoc comparisons. The chi-square test was used to compare categorical variables. The linear regression model was used to predict relationships between nesfatin-1 levels and independent variables. A P value $<0.05$ was considered as statistically significant.

\section{RESULTS}

Table 1 shows the sociodemographic characteristics, plasma nesfatin-1 values, plasma glucose levels, and BMI measurements. There were no significant differences between the three groups in terms of age $(p=0.057)$, gender $(p=0.997)$, tobacco use $(p=0.387)$, and marital status $(p=0.825)$. There were significant differences in 
terms of BMI between the three groups $(p<0.001)$ (Table 1).

Table 1. Baseline characteristics of the study groups

\begin{tabular}{|c|c|c|c|c|}
\hline & Obese & Obese & Non-obese & \\
\hline & + & + & + & $p$ \\
\hline & anxious & non-anxious & non-anxious & \\
\hline & $(n=29)$ & $(n=29)$ & $(n=25)$ & \\
\hline Age (year)a & $45.9 \pm 10.1$ & $46.7 \pm 11.0$ & $40.2 \pm 10.6$ & 0,057 \\
\hline BMI $(\mathrm{kg} / \mathrm{m} 2)^{\mathrm{a}}$ & $37.2 \pm 5.4$ & $38.3 \pm 5.5$ & $22.4 \pm 2.1$ & $<0.001^{b}$ \\
\hline Glucose $(\mathrm{mg} / \mathrm{dL})^{\mathrm{a}}$ & $101.7 \pm 11.5$ & $104.0 \pm 9.1$ & $92.7 \pm 9.4$ & $<0.001^{b}$ \\
\hline Nesfatin $(\mathrm{ng} / \mathrm{mL})^{\mathrm{a}}$ & $28.4 \pm 7.1$ & $28.7 \pm 6.9$ & $34.6 \pm 10.9$ & $0.014^{b}$ \\
\hline Gender (female) & $79.3 \%$ & $79.3 \%$ & $80.0 \%$ & 0,997 \\
\hline Smoking (yes) & $20.7 \%$ & $10.3 \%$ & $24.0 \%$ & 0,387 \\
\hline Marriage (yes) & $89.7 \%$ & $86.2 \%$ & $84.0 \%$ & 0,825 \\
\hline
\end{tabular}

${ }^{a}$ Mean \pm standard deviation, ${ }^{b}$ Statistically significant, BMI: Body Mass Index.

In comparison, there were significant differences between the control group and both patient groups (obese+nonanxious and obese+anxious) $(p<0.001)$ (Table 2 ). Similarly, there were significant differences in terms of serum glucose levels between the three groups $(p<0.001)$ (Table 1).In comparison, there was a significant difference between the control group and both patient groups $(p=0.004$ for obese+anxious group and $p=<0.001$ for obese+non-anxious group) (Table 2 ).
Table 2. Pairwise comparisons of the BMI, glucose, and nesfatin-1 levels between the study groups

\begin{tabular}{|c|c|c|c|}
\hline & Obese & Obese & Non-obese \\
\hline & + & + & + \\
\hline & anxious & non-anxious & non-anxious \\
\hline \multicolumn{4}{|l|}{ BMI } \\
\hline Obese+anxious & 1 & 0,667 & $<0.001^{b}$ \\
\hline Obese+non-anxious & 0,667 & 1 & $<0.001^{b}$ \\
\hline Non-obese+non-anxious & $<0.001^{b}$ & $<0.001^{b}$ & 1 \\
\hline \multicolumn{4}{|l|}{ Glucose } \\
\hline Obese+anxious & 1 & 0,676 & $0.004 b$ \\
\hline Obese+non-anxious & 0,676 & 1 & $<0.001^{b}$ \\
\hline Non-obese+non-anxious & $0.004^{b}$ & $<0.001^{b}$ & 1 \\
\hline \multicolumn{4}{|l|}{ Nesfatin-1 } \\
\hline Obese+anxious & 1 & 0,988 & $0.023^{b}$ \\
\hline Obese+non-anxious & 0,988 & 1 & $0.034^{b}$ \\
\hline Non-obese+non-anxious & $0.023^{b}$ & $0.034^{b}$ & 1 \\
\hline
\end{tabular}

Table 3 shows the relationship between age, HADS scores, BMI measurements, and plasma nesfatin-1 levels. Plasma nesfatin-1 levels were found to be significantly different when compared in the three groups $(p=0.014)$ (Table 1$)$. In posthoc comparisons, plasma nesfatin-1 levels were significantly lower in obese and anxious subjects than in controls $(p=0.023)$, as well as in obese and non-anxious subjects than in controls $(p=0.034)$. There were no significant differences between the obese+anxious group and the obese+nonanxious group $(p=0.988)$.
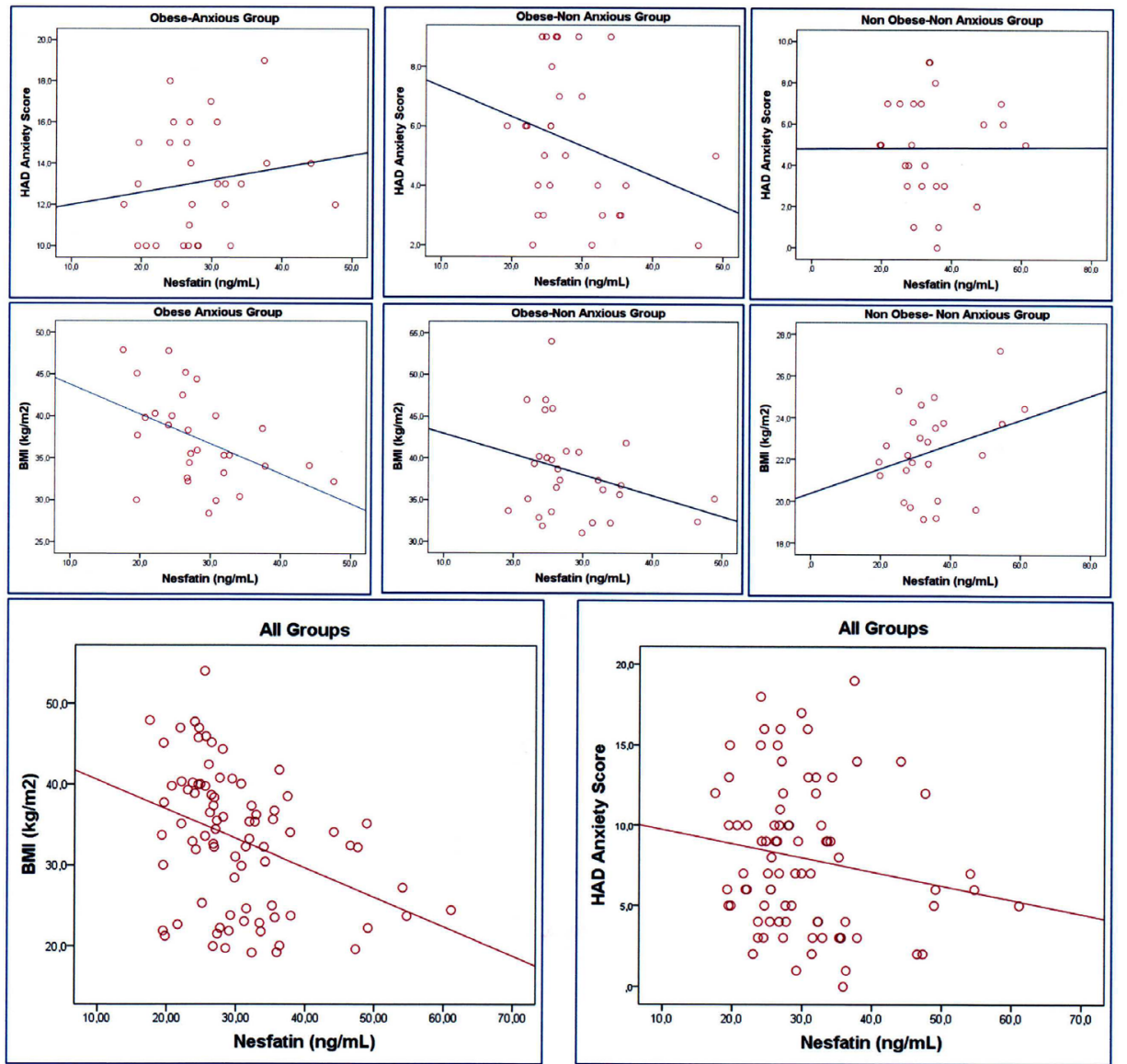

Fig 1. The spesific anxiety scores visualized by histogram graphic. Distrubition of spesific anxiety scores in the groups were reflected. 
Table 3. Correlations between age, HAD anxiety scores, $B M I$, and plasma nesfatin-1 levels

\begin{tabular}{|c|c|c|c|c|c|}
\hline & & Age & $\begin{array}{l}\text { Nesfatin-1 } \\
\text { Levels }\end{array}$ & BMI & $\begin{array}{l}\text { HAD Anxiety } \\
\text { Scores }\end{array}$ \\
\hline \multirow[t]{2}{*}{ Age } & $r$ & 1 & 0,074 & 0,164 & 0,148 \\
\hline & $P$ value & & 0,508 & 0,139 & 0,180 \\
\hline \multirow[t]{2}{*}{ Nesfatin-1 Levels } & $r$ & & 1 & $-0,372$ & $-0,168$ \\
\hline & $P$ value & & & 0,001 & 0,129 \\
\hline \multirow[t]{2}{*}{ BMI } & $r$ & & & 1 & 0,359 \\
\hline & $P$ value & & & & 0,001 \\
\hline \multirow[t]{2}{*}{ HAD Anxiety Scores } & $r$ & & & & 1 \\
\hline & $P$ value & & & & \\
\hline
\end{tabular}

The multivariable linear regression model was used to identify the associations between age, HADS scores, BMI measurements, and plasma nesfatin-1 levels. The regression models were visualized by correlation graphics (Fig. 1). BMI has been identified as an independent variable in the prediction of plasma nesfatin-1 levels $(B=-0.384, p=0.001)$, whereas age and HADS scores were not found to be associated with predicting nesfatin- 1 levels $(B=-0.139, p=0.100$ for age and $B=0.113, p=0.592$ for HADS scores). Fig. 2 shows the specific anxiety scores as visualized by box plots.

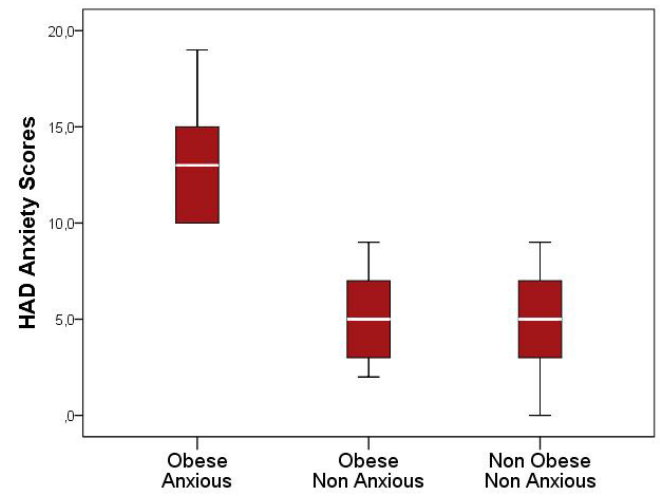

Fig 2. The regressions models visualized by correlation graphics

\section{DISCUSSION}

In our study, we have found a negative relationship between plasma nesfatin-1 levels and BMI. In line with current data, our results support that nesfatin-1 levels may be involved in the development of metabolic abnormalities and obesity. Although studies regarding nesfatin-1 levels in obesity are confounding, our results are consistent with data reporting a negative correlation between nesfatin levels and BMI (8, $22,23)$. Animal studies showed that central administration of nesfatin-1 has been associated with decreased food intake, as well as increased anxiety - and fear-enhancing effects (9). Anxiety-related functions of nesfatin-1 levels have been described not only by directional effects but also by interrelational pathways in the CNS. Similar to nesfatin-1, the corticotropin-releasing hormone $(\mathrm{CRH})$ is one of the peptides with anorexigenic and anxiogenic effects that is abundantly found in the hypothalamic region. Recent studies found that nesfatin-1 and CRH act in a collaborative and serial manner, as $\mathrm{CRH}$ expression is influenced by the hypothalamic nesfatin-1 neurons (9). Activation of the melanocortin system, which has been shown to regulate food intake and anxiety-related behaviors, has also been assumed to be involved in the anxiogenic effects of nesfatin-1 (24). Nesfatin-1 has also been associated with the inhibition of neuropeptide $\mathrm{Y}$, which has anxiolytic effects (25). These experimental studies support the association between anxiety and nesfatin-1. Moreover, in a human study, plasma nesfatin-1 levels have been positively correlated to levels of anxiety in obese populations (26). However, there is some evidence to support that low nesfatin-1 levels are associated with anxiety disorders (15), whereas some studies report no relationship (16). Additionally, a study conducted on obese subjects found a sex-specific, positive correlation between plasma nesfatin-1 levels and anxiety in favor of the female population (27). In a recent study, a sex-specific inverse correlation was observed between NUCB2/nesfatin and anxiety levels, whereas there was no significant change in NUCB2/nesfatin following improvement of anxiety (28). However, our results showed no association between anxiety and plasma nesfatin levels in obese populations. Although our samples were predominantly women, no relationship was observed between anxiety and plasma nesfatin-1 levels. The association between nesfatin-1 levels and anxiety is a complicated issue that needs to take several factors into consideration. For instance, the peripheral measurement of a central acting peptide may not be sufficient to determinate its central role. As animal and clinical studies indicated that central nesfatin- 1 is involved in the regulation of emotional and stress responses, results of the studies that measured peripheral nesfatin- 1 levels in psychiatric conditions are confounding $(9,15,16,29)$. In one study, the authors suggested that nutritional status can be associated with different expressions of nesfatin-1 levels in psychiatric populations (15). Although nesfatin-1 has been shown to have the capacity to cross the blood-brain barrier, the effect of peripheral nesfatin-1 on the CNS or interaction between central and peripheral nesfatin-1 has not been well established. However, as studies focused on peripheral measurements of other regulatory neuropeptides, such as neuropeptide $Y$ and oxytocin, which are involved in central processes and have been related to mood disorders and stress responses $(30,31)$, future studies are required to clarify the association between peripheral nesfatin-1 levels and anxiety disorders, as well as stress-related responses. There are some limitations to our study. First, the sample size was small. Second, individual factors, such as feeding habits and sedentary lifestyles, have not been taken into consideration during the study. Only performing peripheral measurements of nesfatin-1 levels is also a limitation of the study.

\section{CONCLUSION}

In conclusion, our results suggest that peripheral nesfatin-1 levels are not associated with anxiety levels in obese populations. Further studies with a larger sample size are warranted to determine whether there is an association between anxiety and peripheral nesfatin-1 levels. 


\section{REFERENCES}

[1] Stengel A, Mori M, Tache Y. The role of nesfatin-1 in the regulation of food intake and body weight: recent developments and future endeavors. Obes Rev 2013; 14:859870.

[2] Stengel A, Tache Y. Role of brain NUCB2/nesfatin-1 in the regulation of food intake. Curr Pharm Des 2013; 19:6955-6959.

[3] Maejima Y, Kumamoto K, Takenoshita S, Shimomura K. Projections from a single NUCB2/nesfatin-1 neuron in the paraventricular nucleus to different brain regions involved in feeding. Brain Struct Funct 2016; 221:4723-4731.

[4] Stengel A, Hofmann T, Goebel-Stengel M, Lembke V, Ahnis A, Elbelt U, Lamrecht NWG, Ordemann J, Klapp BF, Kobelt P.Ghrelin and NUCB2/nesfatin-1 are expressed in the same gastric cell and differentially correlated with body mass index in obese subjects. Histochem Cell Biol 2013; 139:909-918.

[5] Ramanjaneya M, Addison M, Randeva HS. Possible role of NUCB2/ nesfatin-1 in adipogenesis. Curr Pharm Des 2013; 19:6976-6980.

[6] Ayada C, Toru U, Korkut Y. Nesfatin-1 and its effects on different systems. Hippokratia 2015; 19:4-10.

[7] Hofmann T, Ahnis A, Elbelt U, Rose M, Klapp BF, Stengel A. NUCB2/ nesfatin-1 Is Associated with elevated levels of anxiety in anorexia nervosa. PLoS ONE One 10(7): e0132058.

[8] Tsuchiya T, Shimizu H, Yamada M, Osaki A, Oh IS, Ariyama Y, Takahashi H,Okada S,Hashimoto K,Satoh T,Kojima M,Mori M. Fasting concentrations of nesfatin-1 are negatively correlated with body mass index in non-obese males. Clin Endocrinol (Oxf) 2010; 73:484-490.

[9] Merali Z, Cayer C, Kent P, Anisman H. Nesfatin-1 increases anxiety - and fear-related behaviors in the rat. Psychopharmacology (Berl) 2008; 201:115-123.

[10] Palasz A, Krzystanek M, Worthington J, Czajkowska B, Kostro K, Wiaderkiewicz R, Bajor G. Nesfatin-1, a unique regulatory neuropeptide of the brain. Neuropeptides 2012; 46:105-112.

[11] Shimizu H, Ohsaki A, Oh IS, Okada S, Mori M. A new anorexigenic protein, nesfatin-1. Peptides 2009; 30:995-998.

[12] Xu YY, Ge JF, Qin G, Peng YN, Zhang CF, Liu XR, Liang LC, Wang $\mathrm{ZZ}$, Chen FH,Li J. Acute, but not chronic, stress increased the plasma concentration and hypothalamic mRNA expression of NUCB2/nesfatin-1 in rats. Neuropeptides 2015; 54:47-53.

[13] Bez Y, Ari M, Ozturk OH, Oktar S, Can Y, Sogut S. Plasma nesfatin-1 level may be associated with disease severity in patients with panic disorder. Bulletin of Clinical Psychopharmacology 2010; 20:288-292.

[14] Bez Y, Ari M, Ozturk OH, Oktar S, Can Y. Increased plasma nesfatin-1 levels in patients with obsessive compulsive disorder. Bulletin of Clinical Psychopharmacology 2012; 22:59.

[15] Gunay H, Tutuncu R, Aydin S, Dag E, Abasli D. Decreased plasma nesfatin-1 levels in patients with generalized anxiety disorder. Psychoneuroendocrinology 2012; 37:1949-1953.

[16] Bahceci B, Bagcioglu E, Dilek AR, Celik FH, Bahceci I, Gonul $Y$, Hocaoğlu C.Is There any association between nesfatin-1 and generalized anxiety disorder? Bulletin of Clinical Psychopharmacology 2014; 24:55-58.

[17] Ari M, Ozturk OH, Bez $\mathrm{Y}$, Oktar S, Erduran D. High plasma nesfatin-1 level in patients with major depressive disorder. Prog Neuropsychopharmacol Biol Psychiatry 2011; 35:497500.

[18] Emul M, Karamustafalioglu N, Kalelioglu T, Genc A, Tasdemir A,Gungor FC, Incir S,Seven A. The nesfatin 1 level in male patients with manic episode and alterations of nesfatin 1 level after antipsychotic and electroconvulsive treatment. J Affect Disord 2013; 151:849-853.

[19] First MB, Spitzer RL, Gibbon M, Williams JBW. Structured clinical interview for DSM-IV axis I disorders-clinician version (SCID-CV), 1997. American Psychiatric Association Press, Washington DC 2007.

[20] Zigmond AS, Snaith RP. The hospital anxiety and depression scale. Acta Psychiatr Scand 1983; 67:361-370.

[21] Aydemir O, Guvenir T, Kuey L, Kultur S. Validity and reliability of Turkish version of hospital anxiety and depression scale. Turk Psikiyatri Derg 1997; 8:280-287.

[22] Basar O, Akbal E, Koklu S, Kocak E, Tuna Y, Ekiz F, Gültuna S,YIlmaz F.M,Aydoğan T. A novel appetite peptide, nesfatin-1 in patients with non-alcoholic fatty liver disease. Scand J Clin Lab Invest 2012; 72:479-483.

[23] Guo Y, Xing M, Sun W, Yuan X, Dai H, Ding H. Plasma nesfatin-1 level in obese patients after acupuncture: a randomised controlled trial. Acupunct Med 2014; 32:313-317.

[24] Oh S, Shimizu H, Satoh T, Okada S, Adachi S, Inoue K, Eguchi H,Yamamoto M,Imaki T,Hashimoto K,Tsuchiya T,Monden T,Horuguchi K,Yamada M,Mori M. Identification of nesfatin-1 as a satiety molecule in the hypothalamus. Nature 2006; 443:709-712.

[25] Price CJ, Samson WK, Ferguson AV. Nesfatin-1 inhibits NPY neurons in the arcuate nucleus. Brain Res 2008; 1230:99-106.

[26] Hofmann T, Stengel A, Ahnis A, Buße P, Elbelt U, Klapp BF. NUCB2/ nesfatin-1 is associated with elevated scores of anxiety in female obese patients. Psychoneuroendocrinology 2013; 38:2502-2510.

[27] Hofmann T, Elbelt U, Ahnis A, Rose M, Klapp BF, Stengel A. Sexspecific regulation of NUCB2/nesfatin-1: Differential implication in anxiety in obese men and women. Psychoneuroendocrinology 2015; 60:130-137.

[28] Hofmann T, Weibert E, Ahnis A, Obbarius A, Elbelt U, Rose M, et al. Alterations of circulating NUCB2/nesfatin-1 during short term therapeutic improvement of anxiety in obese inpatients.

[29] Psychoneuroendocrinology 2017; 79:107-115.

[30] Goebel M, Stengel A, Wang L, Tache Y. Restraint stress activates nesfatin-1-immunoreactive brain nuclei in rats. Brain Res 2009; 1300:114-124.

[31] Massey SH, Backes KA, Schuette SA. Plasma oxytocin concentration and depressive symptoms: A review of current evidence and directions for future research. 2 Depress Anxiety 2016; 33:316-322.

[32] Ozsoy S, Olguner Eker O, Abdulrezzak U. The Effects of antidepressants on neuropeptide $Y$ in patients with depression and anxiety. Pharmacopsychiatry 2016; 49:26-31. 